\title{
Treatment of Bacterial Vaginosis in Early Pregnancy and its Effect on Spontaneous Preterm Delivery and Preterm Premature Rupture of Membranes
}

Per-Göran Larsson ${ }^{1,2^{*}}$, Georgios Poutakidis ${ }^{3}$, Annsofie Adolfsson ${ }^{4}$, Georgios Charonis ${ }^{5}$, Pasi Bauer ${ }^{1}$, and Linnea Ekström ${ }^{1}$

${ }^{1}$ Department of Obstetrics and Gynaecology, Skaraborgs Hospital, Skövde, Sweden

${ }^{2}$ University of Linköping, Linköping, Sweden

${ }^{3}$ Department of Obstetrics and Gynaecology, Danderyd Hospital, Sweden

${ }^{4}$ School of Health and Medical Sciences, University of Örebro, Örebro, Sweden and University College of Southeast Norway, Tönsberg, Norway

${ }^{5}$ Mitera General and Maternity Hospital, Athens, Greece

*Corresponding author: Per-Göran Larsson, Department of Obstetrics and Gynaecology, Skaraborgs Hospital, Skövde, Sweden, E-mail: p-g.larsson@vgregion.se Received date: Aug 5, 2016; Accepted date: Sep 12, 2016; Published date: Sep 20, 2016

Copyright: (c) 2016 Larsson PG, et al. This is an open-access article distributed under the terms of the Creative Commons Attribution License, which permits unrestricted use, distribution, and reproduction in any medium, provided the original author and source are credited.

\begin{abstract}
Background: This study was conducted to investigate whether screening and treatment of bacterial vaginosis $(\mathrm{BV})$ in early pregnancy reduces the risk of spontaneous preterm delivery or preterm premature rupture of membranes (PPROMs).

Material and methods: Women were screened for BV during their first visit to the maternal health care unit. After the vaginal samples were air dried, they were sent to the gynecological department and were analyzed using Hay/ Ison modified classification. Eligible women were those who lived in Skaraborg County and delivered at Skaraborgs Hospital in Skövde, Sweden. The women were divided into two groups, namely, screened women (with BV or with lactobacilli flora) and unscreened women. Women with BV were offered treatment with vaginal clindamycin.

Results: During 2007-2015, 22,084 deliveries occurred at Skaraborgs Hospital; a total of 6,899 women were screened for BV, out of which $746(10.8 \%)$ had BV flora. Survival analysis showed that women with BV had spontaneous preterm delivery significantly earlier than those with normal lactobacilli flora, even after treatment with vaginal clindamycin (log rank $p=0.01$ ). During the same period, 15,189 deliveries occurred at Skaraborgs Hospital; they were not screened for BV. The survival analysis showed that the unscreened women delivered slightly earlier than the screened women (t-test $p<0.05$ ), with mean delivery days of 239.4-241.7.
\end{abstract}

Conclusion: Even though patients with BV had been treated with clindamycin, they still suffered from an increased risk of spontaneous preterm delivery as compared to women with normal lactobacilli flora. The difference between the screened women and the unscreened women could be attributed to the positive effect of the treatment of BV with clindamycin if it can be assumed that there is the same amount of untreated BV in the unscreened group.

Keywords: Bacterial vaginosis; Preterm delivery; Pregnancy

Abbreviations: BV: Bacterial Vaginosis; PPROM: Preterm Premature Rupture of Membranes; IRDS: Infant Respiratory Distress Syndrome; IUFD: Intrauterine Foetal Death; GBS: Group B Streptococci; UTI: Urinary Tract Infections; BMI: Body Mass Index; SPSS: Statistical Package of the Social Sciences; CI: Confidence Interval; EPN: Ethical Review Board; IUGR: Intra Uterine Growth Retardation; MHC: Maternity Healthcare Clinics.

\section{Introduction}

Bacterial Vaginosis (BV) is defined as an overgrowth of anaerobic bacteria and the lack of normal lactobacilli flora. It has been associated with poor perinatal outcome, and, in particular, preterm birth [1]. Thus, it is suggested that the diagnosis and treatment of BV may reduce the risk of preterm birth and its consequences [1]. Preterm birth is related to severe perinatal complications such as Infant Respiratory Distress Syndrome (IRDS), sepsis, necrotizing enterocolitis, periventricular and intraventricular haemorrhage, periventricular leukomalacia, and cerebral palsy. Preterm birth can be classified into different subgroups related to the following causes: congenital malformations (4.6\%), intrauterine foetal death (IUFD) (2.7\%), multiple gestations (11.6\%), iatrogenic preterm birth (induction of labour because of clinical infection, abruption of placentae, placenta praevia, intrauterine growth retardation, or preeclampsia) $(20.2 \%)$, and spontaneous preterm birth and preterm premature rupture of membranes (PPROMs) (55.2\%) [2]. Some of the spontaneous preterm births are caused by infection, whereas some are genetically predestinated to have an earlier delivery. The earlier the preterm birth occurs, the more pathological it is and the higher the rate of morbidity. Presently, preterm births between week 34 and week 37 are not clinically challenging. Deliveries that occur between these weeks are treated as normal deliveries, and the children of these women are at a very low risk of any complications. However, preterm birth before 32 weeks is of more concern. Further, preterm birth before 23 weeks is regarded as a miscarriage.

The most commonly accepted laboratory diagnostic test for BV is the Nugent criteria. A swab is obtained from the lateral vaginal wall and is rolled on a glass slide. The smears are Gram stained. The 
Citation: Larsson PG, Poutakidis G, Adolfsson A, Charonis G, Bauer P, et al. (2016) Treatment of Bacterial Vaginosis in Early Pregnancy and its Effect on Spontaneous Preterm Delivery and Preterm Premature Rupture of Membranes. Clin Microbiol 5: 259. doi: $10.4172 / 2327-5073.1000259$

Page 2 of 8

number of Lactobacillus morphotypes is calculated, and if they are more than 30 per vision field, a score of 0 will be recorded. If no lactobacilli are observed, a score of 4 will be recorded. If there are more than 30 small Gram-negative rods or cocci (gardnerella morphotypes), a score of 4 will be recorded. The absence of gardnerella morphotypes will result in a score of 0 . A patient with a score of $0-3$ is considered normal, that with a score of 4-6 is considered to be in the intermediate category, and that with a score of 7-10 is diagnosed with BV [3]. In addition, the presence of curved Gram-variable rods (Mobiluncus species morphotypes) will result in the addition of 1-2 points to the score. Nugent's method suffers from some disadvantages. First, the smears are scored by quantification of the different vaginal morphotypes that requires an experienced slide reader, as well as considerable time and skill [4]. Second, quantification of the morphotypes also depends on the area of the microscope, which can differ by more than $300 \%$ between different microscope vision fields [5], particularly in a modern laboratory microscope. A total of 30 gardnerella morphotypes will result in a score of 4 , thereby indicating that the slide shows intermediate BV. The use of a difference microscope with an area that is $300 \%$ larger will require a total of 90 gardnerella morphotypes to achieve the same score. A simpler method was described by Hay et al. [6], in which the vaginal flora is divided into the following different categories: normal (many lactobacilli morphotypes-few gardnerella morphotypes), intermediate BV (equal numbers of lactobacilli and gardnerella morphotypes), and BV (few lactobacilli and many gardnerella morphotypes) florae. Suggestions have also been given to use the term "Claeys' criteria" instead of Hay/ Ison classification [7].

According to a substantial number of authors, BV during pregnancy is associated with poor perinatal outcomes, and, in particular, an increased risk of preterm birth with potential risk for neonatal sequelae attributed to prematurity. Seventeen articles reporting that BV has been treated during early pregnancy have been published. While the conclusions of these studies differ, it should be noted that different drugs have been used to treat BV. These 17 treatment studies have been discussed in over 23 meta-analyses and reviews. Other infections that can increase the risk of preterm delivery are bacteriuria and infection caused by Neisseria gonorrhoea, Chlamydia trachomatis, and Trichomonas vaginalis as well as bacteriuria. Therefore, the opportunity to reduce preterm birth rates can be approached by the treatment strategy of antimicrobial agents for these conditions. $N$. Gonorrhoea and T. vaginalis are rare diseases in Skaraborg County, why screening for these diseases are not done. All pregnant women are screened in their early pregnancy for bacteriuria. In an earlier publication from the same patient's material, we found no correlation between group B streptococci (GBS) in the vagina or urine and BV [8]. The objective of this study is to investigate whether the treatment of $\mathrm{BV}$ with vaginal clindamycin reduces the risk of spontaneous preterm delivery.

\section{Material and Methods}

This is a prospective study on BV during early pregnancy began in 2007 in the Skaraborg region of Västra Götaland in Sweden. All the pregnant women who were registered with the maternity health care clinics in Skaraborg County and who delivered at Skaraborgs Hospital during 2007-2015 were included in this study. Initially, while not all of the 13 maternity health care clinics in Skaraborg were included in this study, all were eventually considered along the course of the study. In Skaraborg County, there are no private hospitals and all maternity health care clinics use the same computerized medical record, Obsterix. Women who visited the maternity health care clinics in Skaraborg and who were admitted to the delivery ward in Skaraborgs Hospital with signs of risk of preterm birth and were then shifted to another delivery department because either they were expected to deliver extremely prematurely (e.g., gestational weeks 23-27), which necessitated the availability of an advanced neonatal ward in a University hospital, or to another hospital because of limited capacity in the neonatal department in Skaraborgs Hospital to take care of a foetus who was less than 32-weeks old were included to our study. On the other hand, women who visited other maternity health care clinics in other parts of Sweden than Skaraborg County but delivered at Skaraborgs Hospital were excluded from this study, as their medical records are not connected to our Obsterix. Fifteen women who did not visit any maternity health care clinic were also excluded from this study because their duration of pregnancy was uncertain. Because non-Swedish-speaking women could not sign the informed consent form, they were considered to belong to the unscreened group. Further, women with prior antibiotic use were not excluded from this study.

Sample kits were distributed to maternity health care clinics, and women were tested for BV on their first visit to a maternity health care clinic, typically between gestational weeks $10-12$, where a vaginal swab sample and a vaginal $\mathrm{pH}$ test were either self-administered or taken by midwives. The vaginal swab sample was transferred to a microscope slide, air dried, and transported to the gynaecological clinic in Skaraborgs Hospital where it was microscopically examined using a phase contrast microscope with a magnification of $400 \mathrm{x}$, according to the modified Hay/Ison criteria for the diagnosis of BV [9]. The Hay/ Ison criteria were developed using Gram-stained smears by oil immersion at a magnification of 1000x; however, because this criteria assesses the type of flora and does not indicate the number of individual bacteria, which is achieved using Nugent's criteria, it is possible to use these criteria with non-stained smears at lower magnification [5]. The vaginal flora is divided into the following three categories: normal (grade 1), intermediate (grade 2), and BV (grade 3) florae, depending on the relative number of lactobacillus morphotypes as compared to gardnerella morphotypes. At least four fields were evaluated in all vaginal samples. All patients with BV were prescribed treatment with vaginal $2 \%$ clindamycin cream once a day for 7 days, and further follow up with the vaginal swab sample was carried out after the 22nd gestational week. Unfortunately, it was impossible to control whether or not the patients actually took the prescribed medicine. The intermediate group is very poorly defined in terms of the risk of preterm birth, and some authors regard pregnancies belonging to this group as high-risk pregnancies and combine the patients of this group with those of the BV group, claiming their vaginal flora to be "abnormal flora" $[10,11]$. The ethical committee did not approve the treatment of the intermediate group with clindamycin. Therefore, this group was added to the group with normal vaginal flora.

A study where we increase the work loud for the maternal healthcare units could no include all women because of high patient inflow and a lack of midwife resources. We therefore divided the women who we had a vaginal sample into the screened group and women without into the unscreened group. All demographic data, including age and parity, were collected from the computer-based Obstetrix medical register. The Obstetrix medical register includes data from both the maternity health care clinic and the delivery ward; however, it does not include data on miscarriages that occurred up to the 22nd gestational week. The women were evaluated for background 
Citation: Larsson PG, Poutakidis G, Adolfsson A, Charonis G, Bauer P, et al. (2016) Treatment of Bacterial Vaginosis in Early Pregnancy and its Effect on Spontaneous Preterm Delivery and Preterm Premature Rupture of Membranes. Clin Microbiol 5: 259. doi: $10.4172 / 2327-5073.1000259$

Page 3 of 8

factors such as their BMI, as calculated on their first visit to the maternity health care unit; parity; previous history of preterm birth; smoking habits at the time of their first visit to the maternity health care unit and three months before pregnancy; history of previous urinary tract infections (UTIs). On the basis of the body mass index (BMI) measurements, taken at the first visit at the maternal health care clinic, the population was distributed into four BMI classes $(\mathrm{BMI}<19$ : underweight, $19<\mathrm{BMI}<25$ : normal weight, $25<\mathrm{BMI}<30$ : overweight, and $\mathrm{BMI}>30$ : obese). The estimated delivery time was calculated using ultrasound scans between pregnancy weeks 16-18.

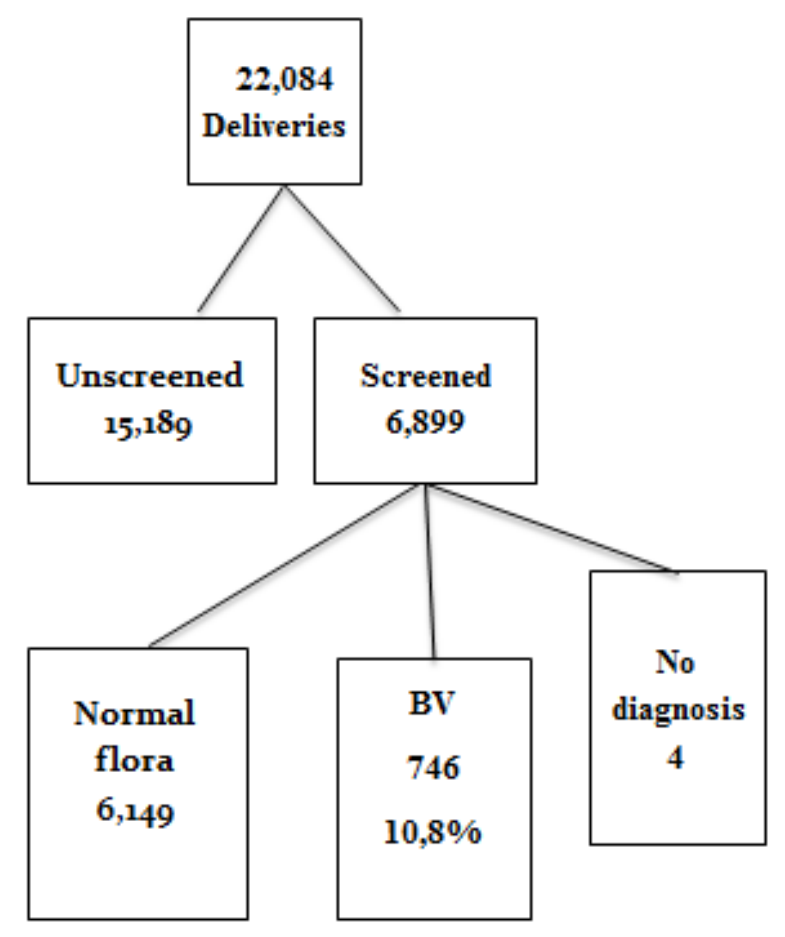

Figure 1: All women who visited the Maternal Health Care clinics in Skaraborg during 2007-2015, and the result of the screening test for bacterial vaginosis $(\mathrm{BV})$.

It is impossible to trace cases related to miscarriages before 23 weeks of gestation because these are not registered in Obstetrix. It was also impossible to include pregnant women who were initially followed up with in the maternity health care units in the region of Skaraborg but shifted to another region in Sweden before delivery. It was possible to classify all the preterm births-defined as those where the patients delivered before the gestational age of 37 weeks and 0 days or 259 days by using the Obstetrix register-into the following categories: intra uterine foetal death (IUFD), multiple gestation, iatrogenic preterm births, spontaneous preterm births, and PPROMs. Iatrogenic preterm birth because it is medically intervened by induction of labour or caesarean section (e.g., preeclampsia, eclampsia, pruritus gravidarum, hepatosis in pregnancy, abruption of placentae, intercurrent disease during pregnancy, symphysiolysis, maternal psychiatric disorders, etc.). Women with spontaneous preterm birth include those whose delivery begin with spontaneous contractions but could end with either vaginal delivery or caesarean section. Women with PPROM could either begin with spontaneous contractions or induced delivery that could either end with vaginal delivery or caesarean section. Multiple gestations have a high risk of preterm delivery, and many of them are induced before 37 weeks of gestation; therefore, we excluded such patients from our analysis.

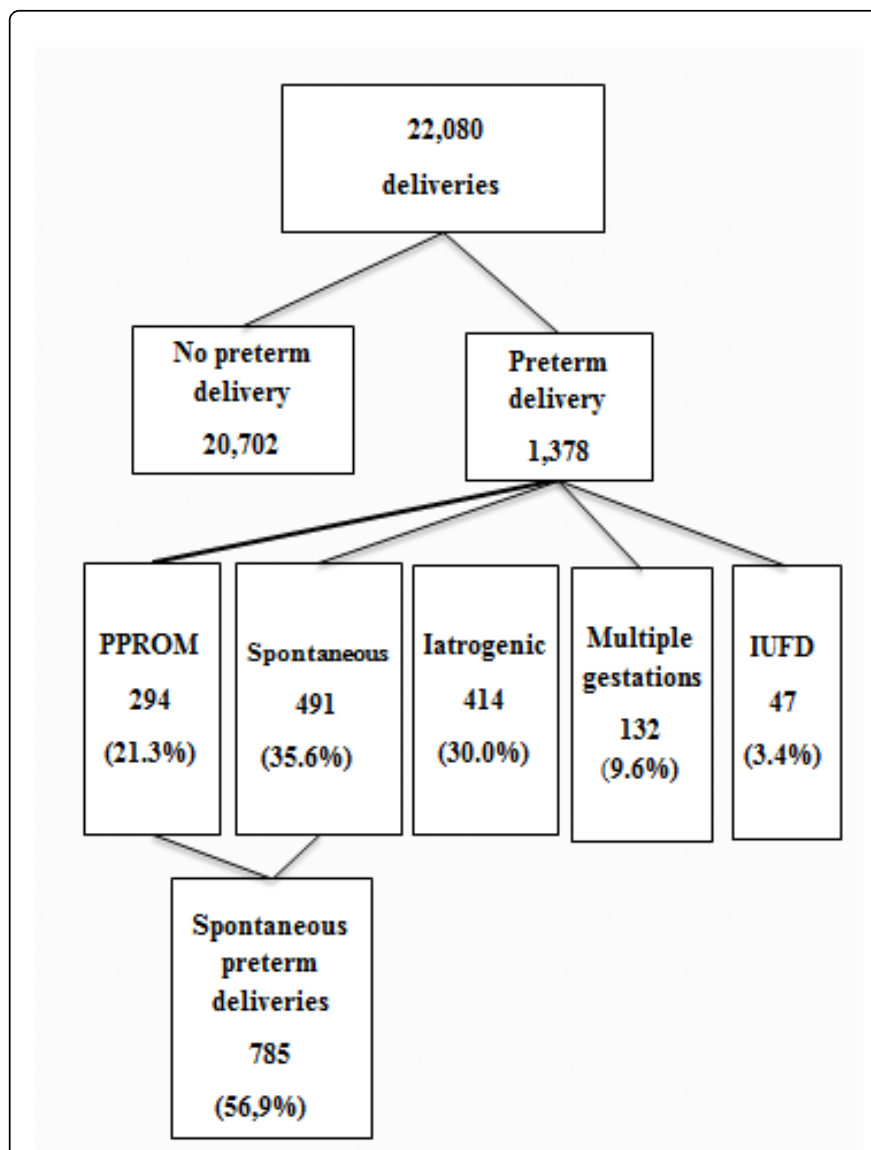

Figure 2: The preterm delivery outcome of all women who delivered at Skaraborgs Hospital during 2007-2015 and those who visited the maternal health care clinics in Skaraborg. It also includes the 91 women who were shifted to other hospitals because of limited capacity at the neonatal department in Skaraborgs hospital.

In our analysis, spontaneous preterm births and PPROM were combined into one group termed the spontaneous preterm birth group. The obtained data were transferred to the Statistical Package for the Social Sciences (SPSS, version 19.0). The statistical methods used were a t-test, odds ratio (OR), and a bivariate analysis using Spearman's rank correlation coefficient. A 95\% confidence interval (CI) was chosen. P-values $<0.05$ were considered significant. Kaplan-Meier survival analysis was also performed to assess the main outcome, which was the gestational age at the time of spontaneous preterm delivery before the 259 th day $(37+0$ gestational week) among the different studied groups and statistical analysis of the log-rank (Mantel-Cox) test. The participants were informed verbally and through a written and signed consent form. Ethical approval was received from the regional ethical review board (EPN) in Gothenburg (2006): number 443-06 and clinical trials number NCT02348463. 
Citation: Larsson PG, Poutakidis G, Adolfsson A, Charonis G, Bauer P, et al. (2016) Treatment of Bacterial Vaginosis in Early Pregnancy and its Effect on Spontaneous Preterm Delivery and Preterm Premature Rupture of Membranes. Clin Microbiol 5: 259. doi: $10.4172 / 2327-5073.1000259$

Page 4 of 8

\section{Results}

The total cohort of pregnant women during the study period 2007-2015 included 22,084 deliveries. A majority of this group was delivered at Skaraborgs Hospital in Skövde. Ninety-one women were shifted to the delivery departments of other hospitals because they entailed the risk of extreme preterm birth (i.e., PPROM, cervical length shortening, intra uterine growth retardation (IUGR), abruption placentae, maternal illness such as severe preeclampsia, or appendicitis before a gestation period of 27 weeks, etc.), which required management at the regional university hospital's perinatal unit or to another hospital because of limited capacity in the neonatal department in Skaraborgs Hospital to take care of a foetus who was less than 32-weeks old. The 22,084 pregnant women were distributed into two groups, namely, those who were screened for BV and those who were unscreened. The numbers of unscreened and screened women were 15,189 and 6,899 , respectively. It was impossible to identify the results of the vaginal specimen of four women; therefore, data obtained for these women were excluded, none of which had a preterm delivery. Thus, 6,895 vaginal samples were to be classified according to the modified Hay/Ison criteria. In the screened group, 746 (10.8\%) women had BV. The intermediate and normal vaginal flora was considered to belong to one group, which was referred to as the group with normal flora consisting of 6,149 individuals (Figure 1).

\section{Distribution of preterm births by cause}

The number of preterm births was 1,376 during the period 2007-2015 at Skaraborgs Hospital, which represents 6.4\% of all the deliveries. This number includes the 91 women that were shifted to another hospital for delivery because of very early gestation (i.e., weeks 23-32). Further, 491 (35.7\%) women were classified to have spontaneous preterm birth, 294 (21.3\%) women were found to suffer from PPROMs, 414 (30.0\%) women had iatrogenic preterm delivery, and $132(9.7 \%)$ women had multiple gestations that that resulted in a preterm delivery. Finally, 47 cases $(3.4 \%)$ were attributed to IUFD (Figure 2). The spontaneous preterm birth and PPROMs were combined, into one group called the spontaneous preterm delivery group, with $782(58.8 \%)$ cases of preterm deliveries.

\section{Population background factors}

In the screened women, there was no difference in the history of preterm delivery between women with BV or with normal vaginal flora. Women with BV were two years younger on an average, were more often nulliparous, had a higher BMI, and smoked more often, both in the three months prior to and during pregnancy (Table 1). The differences between the screened group and the unscreened group are summarized in Table 2. There was no difference in the background factors such as the BMI and a relatively early preterm delivery. However, we found that it was more common for women belonging to the screened group to have a history of earlier UTI's than those belonging to the unscreened group; further, there were a greater number of nulliparous women in the screened group than there were in the unscreened group. It was also found that a greater number of women smoked in the unscreened group than in the screened group (Table 2).

\begin{tabular}{|l|l|l|l|l|l|l|}
\hline Factor & BV (746) & Normal lactobacilli (6149) & OR & Cl 95\% & Missing \\
\hline BMI obese & $138(19.0 \%)$ & $854(14.4 \%)$ & 1.39 & $1.14-1.70$ & P $<0.05$ & 234 \\
\hline Earlier preterm delivery & $58(7.8 \%)$ & $609(9.9 \%)$ & 0.77 & $0.58-1.02$ & N.S. & 0 \\
\hline Previous history UTI & $114(15.5 \%)$ & $986(16.4 \%)$ & 0.94 & $0.76-1.16$ & N.S. & 141 \\
\hline Parity (nullipara) & $402(54.1 \%)$ & $2680(42.8 \%)$ & 1.25 & $1.15-1.35$ & $P<0.05$ & 54 \\
\hline Smoking habits 3 months before & $233(31.6 \%)$ & $865(14.3 \%)$ & 2.78 & $2.24-3.30$ & $P<0.05$ & 92 \\
\hline Smoking first visit MHC & $110(15.1 \%)$ & $343(5.7 \%)$ & 2.95 & $2.34-3.71$ & $P<0.05$ & 139 \\
\hline Bacterial Vaginosis (BV); Urinary Tract Infection (UTI); Maternity Healthcare Clinics (MHC) & & & \\
\hline
\end{tabular}

Table 1: Background factors between the BV group and women with normal lactobacilli flora.

\begin{tabular}{|l|l|l|l|l|l|l|}
\hline \multicolumn{1}{|c|}{ Factor } & \multicolumn{1}{|c|}{ Screened (5457) } & \multicolumn{1}{|c|}{ Unscreened (11554) } & \multicolumn{1}{|c|}{ OR } & \multicolumn{1}{|c|}{ Cl 95\% } & \multicolumn{1}{|c|}{ Missing } \\
\hline BMI obese group & $989(14.9 \%)$ & $2189(15.2 \%)$ & 0.98 & $0.91-1.05$ & N.S. & 1041 \\
\hline Earlier preterm delivery & $667(9.7 \%)$ & $1384(9.1 \%)$ & 1.06 & $0.97-1.16$ & N.S. & 22 \\
\hline Previous history UTI & $1100(16.3 \%)$ & $2127(14.3 \%)$ & 1.14 & $1.07-1.22$ & P $<0.05$ & 456 \\
\hline Parity (nullipara) & $3007(44.0 \%)$ & $6136(40.9 \%)$ & 1.14 & $1.07-1.20$ & $P<0.05$ & 253 \\
\hline Smoking habits 3 months before & $1095(16.1 \%)$ & $2708(18.2 \%)$ & 0.89 & $0.83-0.94$ & P $<0.05$ & 406 \\
\hline Smoking first visit MHC & $451(6.7 \%)$ & $1271(8.5 \%)$ & 0.78 & $0.71-0.87$ & P $<0.05$ & 457 \\
\hline Urinary Tract Infection (UTI), Maternity Health Care clinics (MHC) & & & \\
\hline
\end{tabular}

Table 2: Background factors between the screened and the unscreened groups. 
Citation: Larsson PG, Poutakidis G, Adolfsson A, Charonis G, Bauer P, et al. (2016) Treatment of Bacterial Vaginosis in Early Pregnancy and its Effect on Spontaneous Preterm Delivery and Preterm Premature Rupture of Membranes. Clin Microbiol 5: 259. doi: $10.4172 / 2327-5073.1000259$

Page 5 of 8

\section{Survival analysis of the studied groups with spontaneous preterm births and PPROM}

The survival analysis of the duration of pregnancy was performed on women who delivered before the 259th day of gestation. Of the women with spontaneous preterm births, 236 had normal lactobacilli flora and 36 were BV positive. The difference in the duration of pregnancy between the normal and BV groups is statistically significant (log-rank Mantel-Cox) test $\mathrm{p}=0.010$, $\mathrm{t}$-test $\mathrm{p}<0.05$, with mean delivery days of 246.0 and 239.8, respectively; median 251-248), which indicates that the BV-positive pregnant women had a higher risk of spontaneous preterm births, even after being treated with clindamycin vaginal cream after diagnosis (Figure 3). This difference was still significant if it was compensated for smokers with log-rank (Mantel-Cox) test $\mathrm{p}=0.013$ but not with $\mathrm{t}$-test $\mathrm{p}=0.091$, with mean delivery days of 246.6-242.2. In the screened group, binary logistic regression if spontaneous preterm delivery occurred before or after the 238th day (i.e., $34+0$ gestational week) showed that such delivery depended only on the presence of BV and not on other factors such as the BMI, history of earlier preterm delivery, smoking habit, or UTI."

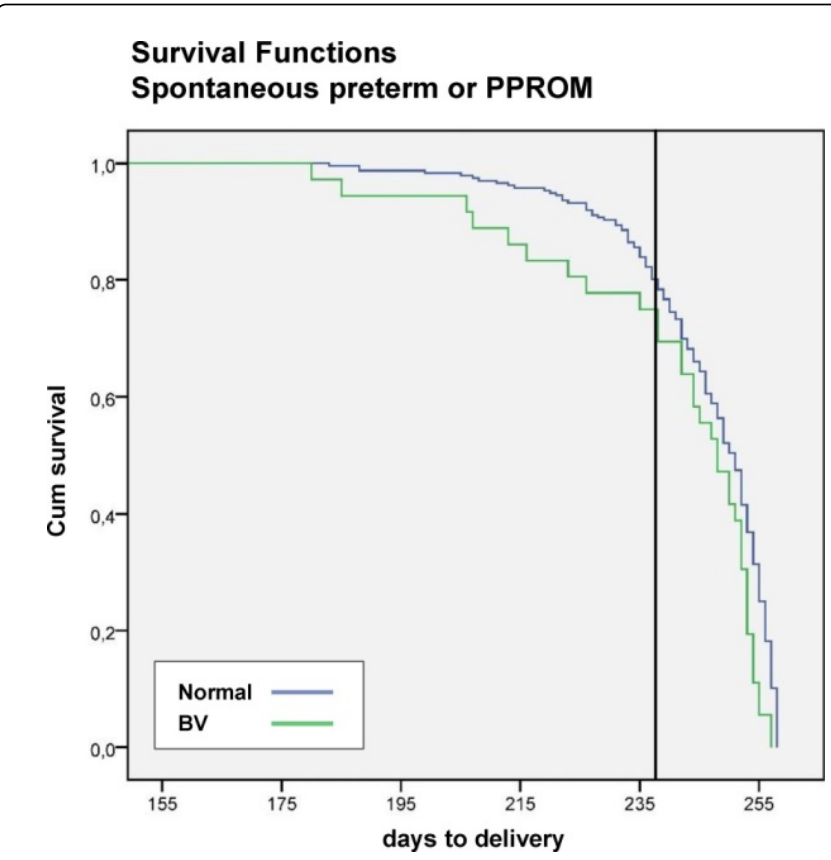

Figure 3: The survival curve (Kaplan-Meier) of spontaneous preterm deliveries or PPROM before the 259th day (37th gestational week and 0 days) shows that the BV-positive women delivered significantly earlier than women with lactobacilli flora even after treatment with clindamycin: log-rank (Mantel-Cox) test $\mathrm{p}<0.05$, t-test $\mathrm{p}<0.05$. The black line indicates the 34 th gestational week.

In order to compare the effect of screening, the unscreened group was compared with the screened group. The survival analysis of spontaneous preterm deliveries or PPROM showed that the unscreened women delivered slightly earlier than the screened women (Figure 4). The result of the log-rank (Mantel-Cox) test is not significant $(\mathrm{p}=0.10)$; however, the result of the $\mathrm{t}$-test $(\mathrm{p}=0.04)$ is significant, with mean delivery days of 239.4-241.7.9. There was a difference between the smoking habits of the unscreened and screened women; a new survival analysis was performed after compensating for smokers, and the difference remained non-significant in the result of both the log-rank (Mantel-Cox) test $(\mathrm{p}=0.085)$ and the t-test $(p=0.056)$, with mean delivery days of 239.9-242.2. Most of the spontaneous preterm deliveries occur after 238th day i.e., after gestational week 34 . The distributions are shown in Figure 5. Only 170 out of 22,084 women had spontaneous preterm delivery or PPROM before day 238, 114 in the unscreened group and 56 in the screened group. The unscreened women delivered at mean day 216 as compared with the screened women who delivered at mean day 222 (t-test: $\mathrm{p}<0.05)$. Nearly all treatments of BV started between the 12th and the 16th week. The survival analysis of treatment that commenced before or after the 14th week shows a slightly better outcome with early treatment especially spontaneous preterm deliveries before the 238th day. However, this difference is not significant ( $\mathrm{t}$-test $>0.05$ ) (Figures 6 and 7).

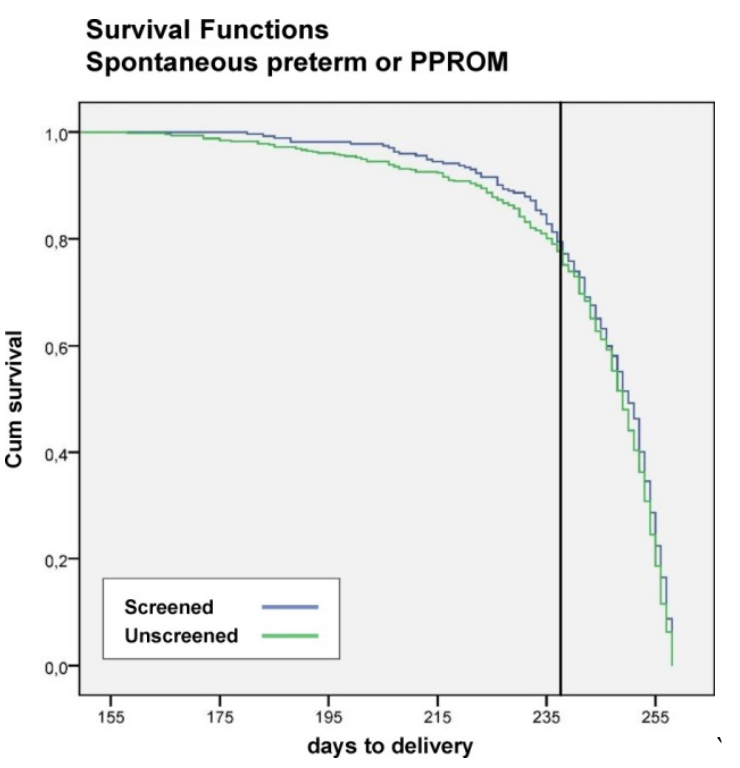

Figure 4: The survival curve (Kaplan-Meier) of spontaneous preterm deliveries or PPROM shows that the unscreened group delivered significantly earlier than the screened group: log-rank (Mantel-Cox) test $\mathrm{p}<0.05$. The biggest difference before day 238 (i.e., before the 34 th gestational week). The black line indicates the 34 th gestational week.

\section{Cure rate of BV after treatment with clindamycin $2 \%$ vaginal cream}

The BV group consisted of 746 women who were treated with clindamycin for one week. Of these women, 222 (29.8\%) women failed to return for follow up after treatment. Only 47 out of 477 women of the controlled group had persistent BV, which had a cure rate of $90.1 \%$ after the first treatment. These $47 \mathrm{BV}$-positive women were offered another week's treatment with clindamycin $2 \%$ vaginal cream; of these women, 22 women failed to return for follow up after treatment. Only 7 out of 25 of those who came back had persistent BV, which has a cure rate of $72 \%$ after the second treatment. The 7 women with relapse of BV were again offered clindamycin treatment, but no controlling measures were taken after treatment. Of the 222 women that did not 
Citation: Larsson PG, Poutakidis G, Adolfsson A, Charonis G, Bauer P, et al. (2016) Treatment of Bacterial Vaginosis in Early Pregnancy and its Effect on Spontaneous Preterm Delivery and Preterm Premature Rupture of Membranes. Clin Microbiol 5: 259. doi: $10.4172 / 2327-5073.1000259$

Page 6 of 8

return for follow up after the treatment of BV, 8 had spontaneous preterm delivery and 5 had iatrogenic preterm delivery (Figure 8 ).

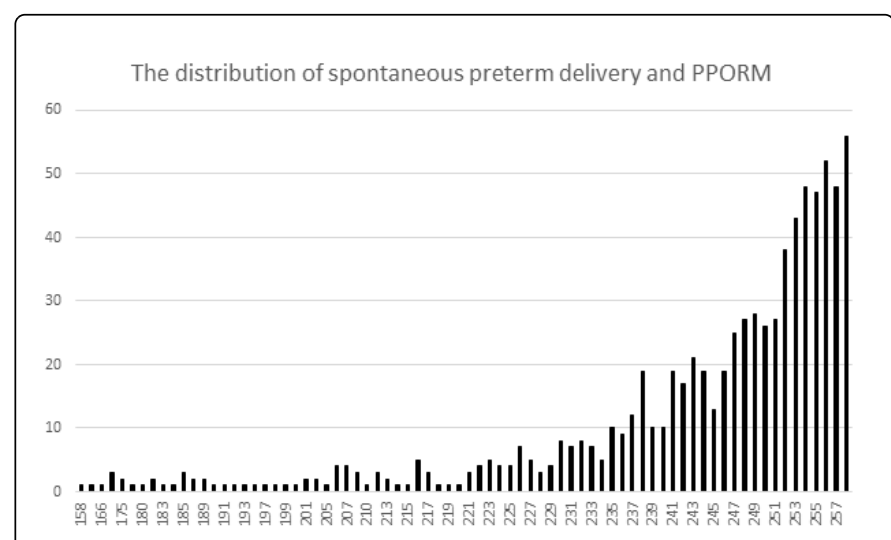

Figure 5: Most of the spontaneous preterm deliveries or PPROM occur after day 238 i.e., after gestational week 34 . Most of those are not because of infection but are rather physiological deliveries. As this is the majority, it is difficult to show statistical significance of treatment infections. The black line indicates the 34th gestational week.

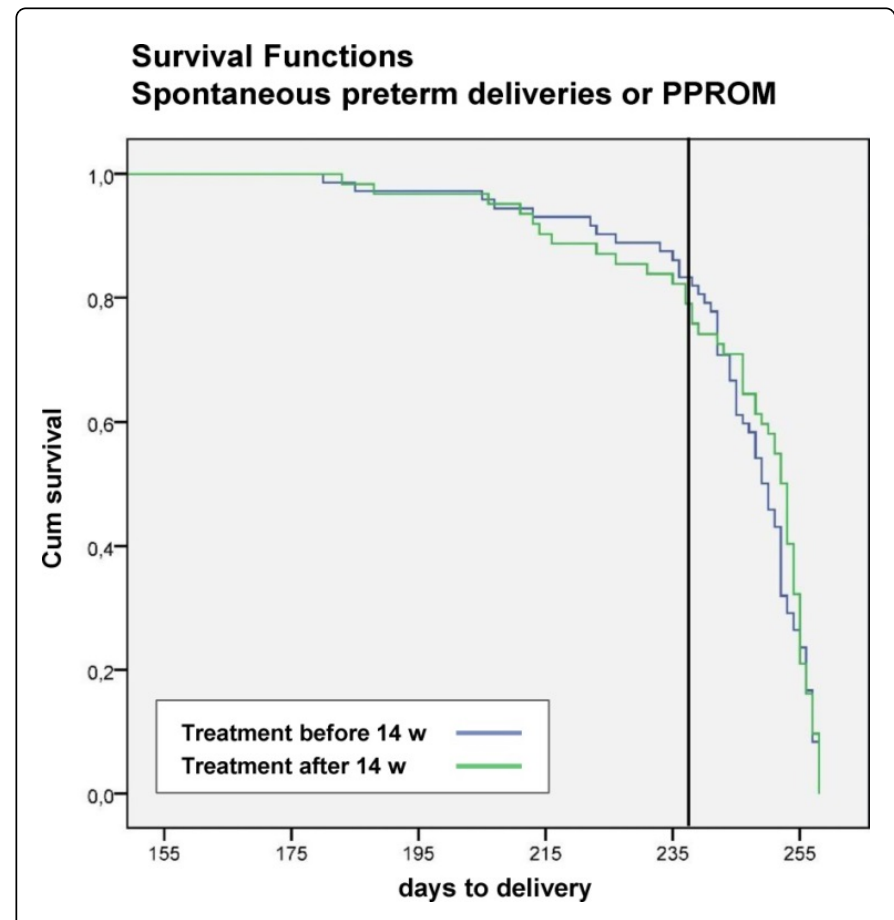

Figure 6: The survival curve (Kaplan-Meier) of spontaneous preterm births or PPROM shows that there was no statistical difference if the treatment commenced before or after the 14th gestational week. The black line indicates the 34 th gestational week.

Of the 47 women with relapse of $\mathrm{BV}$, one had a spontaneous preterm delivery and two had an iatrogenic preterm delivery. None of the 7 women with second relapse of BV had preterm delivery. We investigated whether the rate of preterm delivery decreased during our study period, however, this difference was not significant. However, a trend showing a decrease in the number of preterm deliveries is observed (data not shown). The difference in the BV frequency between different maternity health care clinics in Skaraborg County varies from 5\% to $19 \%$. In Skaraborg, there are 13 different maternity clinics, and only 132 deliveries have been carried out in the smallest maternity clinic. Therefore, the small clinics are merged with the neighbouring clinics giving six different clinics. These clinics represent the clinics in the six bigger towns in the area (Mariestad, Skövde, Falköping, Skara, Lidköping, and Hjo/Karlsborg). These clinics will then be able to accommodate between 2100 and 7000 deliveries. The frequency of BV is likely to be $9.2 \%-12.7 \%$, and the frequency of spontaneous preterm delivery will be between $2.9 \%$ and $3.8 \%$. These differences are non-significant.

\section{Discussion}

This study shows, that the BV group, even if treated with clindamycin, is at a risk of preterm delivery, and, therefore, patients belonging to this group should be provided with extra prenatal care. All former studies dealing with the treatment of BV to reduce the risk of preterm delivery have only compared a treated BV group with an untreated BV group, either with a placebo or without treatment; however, none of the studies have compared women with BV that have been treated with women with normal lactobacilli flora. Our results indicate that women with BV, even after treatment, will suffer from an increased risk of preterm delivery, which might explain some of the controversy regarding whether the treatment of BV during early pregnancy is recommended. When designing a study, one estimates the sample size that is required to show the effects of the treatment. In doing so, one assumes that the treatment of BV will reduce the risk of spontaneous preterm delivery to the same level as that entailed by women without BV.

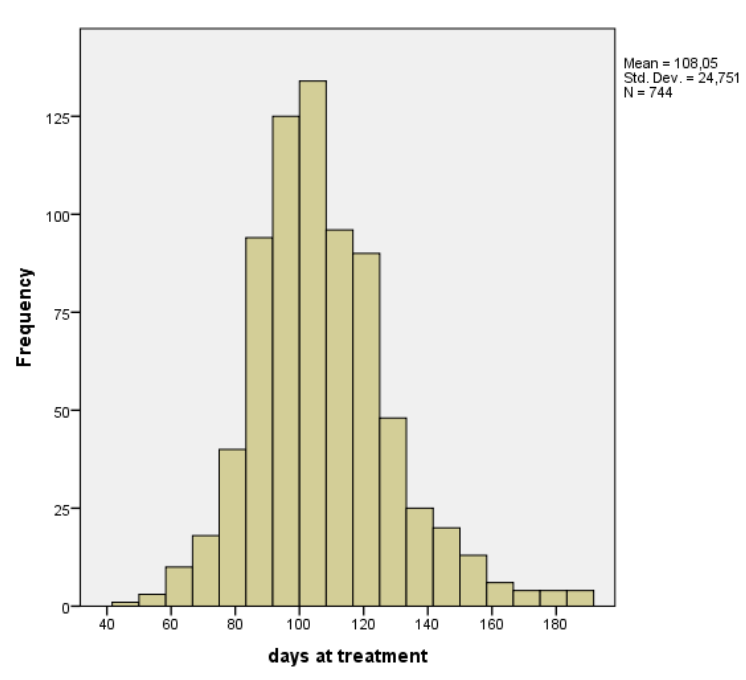

Figure 7: Plot showing the distribution of when the treatment commenced with clindamycin against BV.

The results of our study suggest that while the treatment of BV could reduce the risk of spontaneous preterm delivery, it will not reduce it to the same extent as that for women with lactobacilli flora. Lamont [12] suggested that treatment of BV between the 12th and the 20th gestational week might reduce the risk of preterm delivery by 
Citation: Larsson PG, Poutakidis G, Adolfsson A, Charonis G, Bauer P, et al. (2016) Treatment of Bacterial Vaginosis in Early Pregnancy and its Effect on Spontaneous Preterm Delivery and Preterm Premature Rupture of Membranes. Clin Microbiol 5: 259. doi: $10.4172 / 2327-5073.1000259$

Page 7 of 8

$40 \%$. Therefore, several studies could not provide a significant result with regard to the treatment because they were underpowered.

The existing literature regarding the treatment of $\mathrm{BV}$ is very inconsistent. Some authors argue that it is important to commence the treatment as early as possible. A majority of the studies show a statistically significant association between BV and preterm birth and that the risk of preterm birth was higher if BV was diagnosed in the early pregnancy [12]. If antibiotics are used late in the pregnancy when inflammatory tissue damage may have already occurred, then antibiotics are unlikely to be beneficial and may even have detrimental effects. In our study, we chose to commence most of the treatment after the 12th week. However, we could not show whether the treatment before or after the 14th week made any difference. Most treatments in our study commenced between the 12th and the 16th week. A systematic review by Lamont et al. of clindamycin used in women with abnormal flora, including BV, in the early pregnancy before 22 weeks of gestation was published in [13]. It was hypothesized that the conclusions of individual studies/systematic reviews/meta-analyses on the use of antibiotics used prophylactically for the prevention of preterm birth were flawed by the following factors:

1. suboptimal choice of antibiotics,

2. antibiotics used too late in the pregnancy to influence the outcome (23-27 weeks), and

3. antibiotics used in women whose risk of preterm birth was not attributed to BV.

In this study, we attempted to avoid these three factors. Because we could not treat the intermediate group according to the ethical committee, we combined the intermediate group with the group with normal vaginal flora.

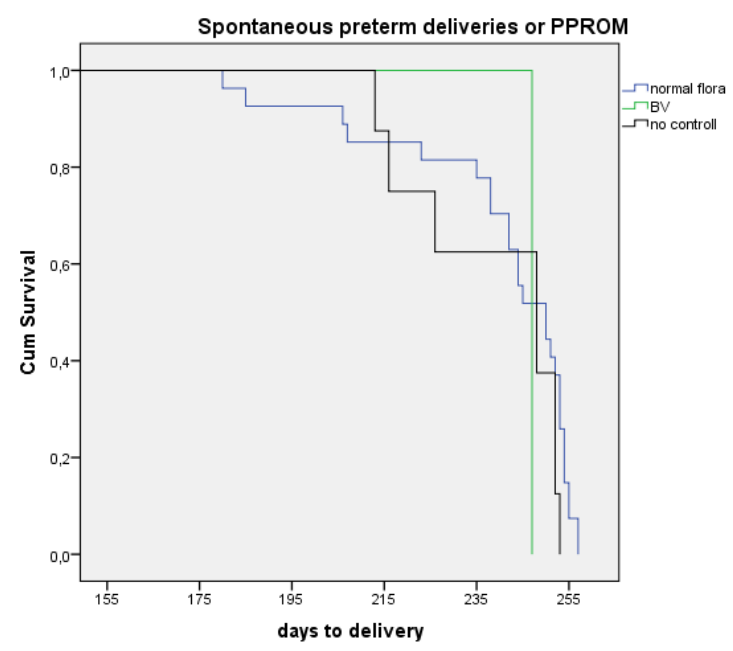

Figure 8: The survival curve (Kaplan-Meier) of spontaneous preterm deliveries or PPROM among women treated for BV divided between the ones that were cured, not cured, and did not return for follow up. This curve shows that the women who did not return for follow up did not deliver earlier than women that where cured for the BV. Only one woman who had a persistence of BV had a spontaneous preterm delivery. The black line indicates the 34th gestational week.
The distribution of the preterm births in this relatively large prospective study was found to be similar to the distribution reported in previous papers [2]. The prevalence of BV in the screened group was $10.8 \%$, which is comparable to the result reported by Desseauve et al. [14]. In an earlier study, we have showed that the treatment of BV during early gestation is likely to prolong the pregnancy by 30 days [15]. In that study, all miscarriages that occurred after the 16th week of gestation were included. Unfortunately, it was impossible for us to find out how many miscarriages occurred at our hospital from our medical record. We use another computerized medical system for gynaecological diseases which include all miscarriage (Melior). This diagnosis (ICD10) does not differentiate between early and late miscarriage. If the $\mathrm{BV}$ treatment prolongs the pregnancy by 30 days, a miscarriage that would have occurred in the 21 st week if untreated could then become a preterm delivery in the 25 th week if treated. This problem will be very difficult to solve with a computerized journal system-Obstetrix-which only registered the children born after the 23rd week. While designing a new study, one has to take into consideration the late miscarriages that occur between gestational weeks 16 and 23. This study was however not designed to investigate women who had a late miscarriage. We did a thoughtful survey of all the miscarriages that occurred during 2013 (unpublished results). We identified 720 miscarriages, 16 of which occurred after the 16th week. If this result could be extrapolated to the entire study period, it would imply that approximately 110 late miscarriages should occur during this time. As only 170 spontaneous preterm deliveries occur before the 34 th gestational week, late miscarriages could be of great importance. During the first years of the study, we could identify late miscarriages in the screened group. Of the 21 women who suffered from late miscarriages in the screened group, only 2 were BV positive. Because we had no possibility to find out the total number of late miscarriages in the unscreened group, we stopped recording the instances of late miscarriages even in the screened group.

It is interesting to note that the unscreened women had significantly more spontaneous preterm deliveries than the screened women. In this group, we can expect that there are as many women with BV (and these should then not be treated) as there are in the screened group. If so, this difference will then be the effect of treatment with clindamycin. To do so, we have to assume that the screened and the unscreened populations entail the same risk factors as the screened group. However, we know that the early occurrence of UTI is significantly more common in the screened group than in the unscreened group; further, smokers are significantly more common in the unscreened group than in the screened group. If women who smoked were excluded, the difference in spontaneous preterm delivery was not significant as the p-value was increased from 0.040 to 0.056 if the smokers were excluded. If we had not investigated the unscreened group, the first conclusion of our study would have been that the treatment of BV with clindamycin would not be beneficial or that the treatment was doing more harm than good. It is also interesting to note that the biggest difference between the screened and the unscreened groups lies in the earlier gestational weeks. This is the most clinically challenging aspect as clinician's will attempt to prolong the pregnancy with anti-contraction drugs and antibiotics combined with corticosteroids that help to mature the lungs of the infant. Many of the studies that have debated the treatment of BV during pregnancy have only had a primary objective to investigate the number of deliveries before the 259th day (37 weeks and 0 day). Many of the deliveries between the 238th and the 259th day are not caused by an infection but are instead a physiological delivery (Figure 5). This is the most 
Citation: Larsson PG, Poutakidis G, Adolfsson A, Charonis G, Bauer P, et al. (2016) Treatment of Bacterial Vaginosis in Early Pregnancy and its Effect on Spontaneous Preterm Delivery and Preterm Premature Rupture of Membranes. Clin Microbiol 5: 259. doi: $10.4172 / 2327-5073.1000259$

Page 8 of 8

clinically significant finding, as children who are born before 238th day are at a greater risk of sequelae than children born after 238 days. If the treatment of BV with clindamycin will save only a few spontaneous deliveries between day 180 and day 220 (gestational weeks 26-32), the rate of morbidity will be significantly reduced. Only 170 spontaneous preterm deliveries occurred before the 238th day $(34+0$ gestational week); thus such births are extremely rare even in a large population as ours that consist of 22,084 women. We also found that these very early preterm deliveries occurred nearly one week earlier in the unscreened group as compared with the screened group $(\mathrm{p}<0.05)$. A difference of one week could be very significant in these gestational weeks.

A disadvantage of our study is that only around $30 \%$ of the women were screened because of high patient inflow and a lack of midwife resources at the maternal healthcare units. The social surroundings and the inhabitants have very similar maternal healthcare units, and all the cities are small with only around 30,000 inhabitants. There is little difference in the frequency of BV and preterm delivery between the six small towns; therefore, we can only assume that the screened and unscreened populations are similar, even if we cannot confirm this assumption. Another disadvantage of our study is that we have no treatment control of as many as 222 of 746 women treated for BV. However, according to the Kaplan-Meier curve shown in Figure 8, women who were not controlled after the treatment of BV will not deliver earlier than those who were cured after treatment of BV. A test to determine the cure rate was scheduled to be performed 10-15 weeks (after gestational week 22) after the treatment. While designing a new study, a test to determine the cure rate should be performed after 4 weeks.

Another aspect to consider when doing a new study would to investigate what lactobacilli flora the women has after treatment of BV and to study if addition of probiotic lactobacilli will lower the risk of preterm delivery. Petricevic at al. have shown that women with Lactobacillus iners have a greater risk of preterm delivery than women with other types of lactobacillus spp. [16]. He has also shown that different lactobacillus can restore the vaginal flora after antibiotic treatment of BV [17]. In the latest review by Haahr el al. [18] just published they still look at spontaneous preterm delivery before 37th week and the worst error is that they add a study by Subtil that are unpublished and are only available only as an abstract. This study will interfere with the result and make the conclusion of the review not to recommend treatment of $\mathrm{BV}$.

\section{Conclusion}

To the best of our knowledge, this is the first study that compares women with BV who have been treated with clindamycin with women with normal lactobacilli flora in terms of spontaneous preterm birth. Women with BV, even when treated, still have a significantly higher risk of spontaneous preterm birth than women with lactobacilli flora. The advantage of treating BV could explain the difference in the gestational age during preterm spontaneous delivery between the screened and the unscreened women.

\section{Acknowledgement}

This study had received funding from Skaraborgs hospital FoU fund.

\section{References}

1. Leitich H, Kiss H (2007) Asymptomatic bacterial vaginosis and intermediate flora as risk factors for adverse pregnancy outcome. Best Pract Res Clin Obstet Gynaecol 21: 375-390.

2. Morken NH, Källen K, Hagberg H, Jacobsson B (2005) Preterm birth in Sweden 1973-2001: rate, subgroups, and effect of changing patterns in multiple births, maternal age, and smoking. Acta Obstet Gynecol Scand 84: 558-565.

3. Nugent RP, Krohn MA, Hillier SL (1991) Reliability of diagnosing bacterial vaginosis is improved by a standardized method of gram stain interpretation. J Clin Microbiol 29: 297-301.

4. Chawla R, Bhalla P, Chadha S, Grover S, Garg S (2013) Comparison of Hay's criteria with Nugent's scoring system for diagnosis of bacterial vaginosis. Biomed Res Int 2013: 365194.

5. Larsson PG, Carlsson B, Fåhraeus L, Jakobsson T, Forsum U (2004) Diagnosis of bacterial vaginosis: need for validation of microscopic image area used for scoring bacterial morphotypes. Sex Transm Infect 80: 63-67.

6. Hay PE, Lamont RF, Taylor-Robinson D, Morgan DJ, Ison C, et al. (1994) Abnormal bacterial colonisation of the genital tract and subsequent preterm delivery and late miscarriage. BMJ 308: 295-298.

7. Verhelst R, Verstraelen H, Claeys G, Verschraegen G, Van Simaey L, et al. (2005) Comparison between Gram stain and culture for the characterization of vaginal microflora: definition of a distinct grade that resembles grade I microflora and revised categorization of grade I microflora. BMC Microbiol 5: 61.

8. Ekström L, Adolfsson A, Ericson H, Poutakidis P, Charonis G, et al. (2013) Vaginal flora and urinary and vaginal group B streptococci in early pregnancy. Gynecology.

9. Ison CA, Hay PE (2002) Validation of a simplified grading of Gram stained vaginal smears for use in genitourinary medicine clinics. Sex Transm Infect 78: 413-415.

10. Lamont RF, Taylor-Robinson D (2010) The role of bacterial vaginosis, aerobic vaginitis, abnormal vaginal flora and the risk of preterm birth. BJOG 117: 119-120.

11. Donders GG, Riphagen I, van den Bosch T (2000) Abnormal vaginal flora, cervical length and preterm birth. Ultrasound Obstet Gynecol 16: 496-497.

12. Lamont RF, Taylor-Robinson D, Bassett P (2012) Rescreening for abnormal vaginal flora in pregnancy and re-treating with clindamycin vaginal cream significantly increases cure and improvement rates. Int $\mathrm{J}$ STD AIDS 23: 565-569.

13. Lamont RF, Nhan-Chang CL, Sobel JD, Workowski K, Conde-Agudelo A, et al. (2011) Treatment of abnormal vaginal flora in early pregnancy with clindamycin for the prevention of spontaneous preterm birth: a systematic review and metaanalysis. Am J Obstet Gynecol 205: 177-190.

14. Desseauve D, Chantrel J, Fruchart A, Khoshnood B, Brabant G, et al. (2012) Prevalence and risk factors of bacterial vaginosis during the first trimester of pregnancy in a large French population-based study. Eur J Obstet Gynecol Reprod Biol. 163: 30-34.

15. Larsson PG, Fåhraeus L, Carlsson B, Jakobsson T, Forsum U, et al. (2006) Late miscarriage and preterm birth after treatment with clindamycin: a randomised consent design study according to Zelen. BJOG 113: 629-637.

16. Petricevic L, Domig KJ, Nierscher FJ, Sandhofer MJ, Fidesser M, et al. (2014) Characterisation of the vaginal Lactobacillus microbiota associated with preterm delivery. Sci Rep 4: 5136.

17. Petricevic L, Witt A (2008) The role of Lactobacillus casei rhamnosus Lcr35 in restoring the normal vaginal flora after antibiotic treatment of bacterial vaginosis. BJOG 115: 1369-1374.

18. Haahr T, Ersbøll AS, Karlsen MA, Svare J, Sneider K, et al. (2016) Treatment of bacterial vaginosis in pregnancy in order to reduce the risk of spontaneous preterm delivery - a clinical recommendation. Acta Obstet Gynecol Scand 95: 850-860. 